\title{
Comunicación
}

\section{Reporte de Adenocarcinoma Pulmonar Ovino en un Cordero de Cinco Meses de Edad en Puno, Perú}

\author{
Report of an Ovine Pulmonary Adenocarcinoma in a Lamb of Five Months Old \\ in Puno, Peru
}

Pablo Londoñe ${ }^{1}$, Lenin Maturrano H. ${ }^{1}$, Raúl Rosadio A. ${ }^{1,2}$

\section{Resumen}

\begin{abstract}
Se reporta un caso de adenocarcinoma pulmonar ovino en un cordero criollo de cinco meses de edad, procedente del departamento de Puno, Perú. El animal, a la inspección clínica, presentó disnea, pérdida de peso y abundante secreción pulmonar. A la necropsia se observaron formaciones nodulares blanco grisáceas en los lóbulos diafragmáticos y abundante fluido espumoso blanquecino en la tráquea. En la superficie de corte de los tumores se visualizaron numerosos micronódulos de contorno irregular con tendencia a la coalescencia. Histológicamente, las formaciones tumorales, compuestas por células columnares o cuboidales, se mostraron aisladas por un estroma fibrosado y rodeadas por alveolos inundados de macrófagos con citoplasma vacuolar. En los bronquios terminales se observaron pólipos intraluminales comprometiendo el epitelio bronquiolar. El análisis molecular del fluido y tejido pulmonar contenían secuencias genéticas del retrovirus exógeno del Tipo D productor de la enfermedad. La infección retroviral con presencia multinodular de lesiones neoplásicas en un cordero corrobora la susceptibilidad de ovinos criollos a la infección retroviral.
\end{abstract}

Palabras clave: cordero, adenocarcinoma pulmonar ovina, enfermedad de Jaagsiekte

\section{AbSTRACT}

A case of ovine pulmonary adenocarcinoma is reported in a five month-old indigenous lamb of the department of Puno, Peru. At clinical inspection, the animal presented dyspnea, body weight loss and abundant lung secretion. The necropsy revealed in both

${ }^{1}$ Laboratorio de Microbiología y Parasitología Veterinaria, Facultad de Medicina Veterinaria, Universidad Nacional Mayor de San Marcos, Lima Perú

22E-mail: rrosadio@gmail.com

Recibido: 12 de mayo de 2014

Aceptado para publicación: 15 de setiembre de 2014 
diaphragmatic lobes the presence of two grayish white nodular formations with plenty of white foamy fluid emanating from the trachea. At the cut surface of the tumor was observed irregular micronodules with a tendency to coalesce. Histologically, the tumors were composed of columnar or cuboidal cells isolated by a fibrotic stroma and surrounded by alveoli flooded with macrophages of vacuolar cytoplasm. Intraluminal polyps were observed in the terminal bronchioles compromising the bronchiolar epithelium. Molecular analysis of lung tissue and fluid contained the exogenous genetic sequences of type D retrovirus. Retroviral infection with multi nodular presence of neoplastic lesions in a lamb corroborates the susceptibility of indigenous Andean sheep to retroviral infection

Key words: lamb, ovine pulmonary adenocarcinoma, Jaagsiekte's disease

\section{INTRODUCCIÓN}

La adenomatosis pulmonar ovina (APO) es un cáncer pulmonar altamente transmisible, producido por un betaretrovirus oncogénico de semejanza histológica al carcinoma bronquiolar en humanos (Palmarini et al., 1999; De las Heras et al., 2003; Sharp y DeMartini, 2003). La enfermedad, históricamente denominada Jaagsiekte, es también conocida como Adenocarcinoma Pulmonar Ovino. Es de distribución mundial y causa grandes repercusiones económicas en países tales como Escocia, Perú y Sudáfrica (Cuba Caparó et al., 1961; Wandera, 1970; Sharp y DeMartini, 2003).

La enfermedad fue descrita en el Perú en 1945 con características epizoóticas; inicialmente en la sierra central del país (Cuba Caparó et al., 1961; Snyder et al., 1983) y posteriormente diseminada a otras regiones geográficas, producto de intercambios comerciales de reproductores mejorados procedentes de empresas asociativas de la sierra central hacia el sur, donde infectaron a poblaciones de ovinos criollos (Ellis et al., 1993). La APO, en su presentación natural, se observa usualmente en animales adultos (2-4 años de edad) (Tustin, 1969; Hunter y Munro, 1983; González et al., 1993; Cutlip y Young, 1982), pero se han reportado casos esporádicos en corderos de 6 a 9 meses de edad (Sharp y DeMartini, 2003).
En el Perú, los primeros reportes de la enfermedad involucran animales adultos (Cuba Caparó et al., 1961); sin embargo, Paredes (1953) reporta la enfermedad en una empresa lanera de la sierra central, donde 3 de los 20 casos fueron en corderos de 7 meses de edad. La infección fue, asimismo, detectada en un cordero Corriedale de 6 meses, que nació y convivió con su madre experimentalmente infectada, en un ambiente cerrado en el Valle del Mantaro (R. Rosadio, datos no publicados).

Clínicamente, la enfermedad usualmente se expresa con una intensa fatiga, disnea, pérdida progresiva de peso y producción de abundante secreción pulmonar (Sharp y Angus, 1990; De las Heras et al., 2003). Las características patológicas corresponden a formaciones multinodulares de células epiteliales secretoras neumonocito tipo II y células bronquiolares (células de Clara) no ciliadas, pero con un bajo porcentaje de metástasis en los nódulos linfáticos regionales (DeMartini et al., 1988; Rosadio et al., 1988).

La enfermedad es producida por un retrovirus oncogénico exógeno de Tipo D, detectado consistentemente en secreciones pulmonares y en homogenizados tumorales de animales infectados natural o experimentalmente (Rosadio y Zavaleta, 2001). El retrovirus todavía no ha podido ser aislado en el laboratorio; sin embargo, mediante técnicas biomoleculares se ha podido identificar 
secuencias genéticas muy similares (endógenas) que solamente se diferencian de las secuencias patogénicas (exógenas) en la región U3 de los TLR retroviral (Palmarini et al., 1996). Estas han sido utilizadas para diseñar marcadores moleculares específicos para la detección del virus exógeno productor de la enfermedad en tejido neoplásico pulmonar, tejido linfoide y aún en tejido mamario (González et al., 2001).

El presente trabajo reporta la identificación de adenocarcinoma pulmonar ovino en un cordero de la zona altoandina sur del Perú.

\section{Materiales y Métodos}

La identificación del animal afectado ocurrió durante el desarrollo de un proyecto de investigación en el periodo 2002-2003 para identificar presencia molecular en tejidos pulmonares del beta retrovirus productor de la enfermedad. El animal sospechoso, un cordero de raza criolla de 5 meses de edad, fue detectado en una majada de animales criollos en el distrito de Ayaviri, provincia de Melgar, departamento de Puno, Perú.

Se obtuvo el permiso para sacrificar el animal y se procedió a realizar la necropsia en el mismo lugar de crianza. Se enfatizó en la cavidad pulmonar, anotándose las alteraciones macroscópicas en un formato preparado para registrar lesiones tumorales. Se tomaron muestras tumorales en frascos con formol al 10\%. Las muestras de pulmón fueron procesadas siguiendo técnicas histológicas convencionales y coloreadas con hematoxilina y eosina. Asimismo, muestras tumorales fueron preservadas en nitrógeno líquido para su análisis molecular.

Se extrajo el ADN de una muestra tumoral mediante un kit de extracción comercial (Quiagen, EEUU) y para la prueba de PCR semi-anidada se utilizaron cebadores específicos (PI y PIII en la primera y PI y
PIV en la segunda ronda) diseñadas para detectar el ADN proviral en la región U3 del TLR del retrovirus exógeno productor de la enfermedad, siguiendo la metodología descrita por Palmarini et al. (1996).

\section{Resultados y Discusión}

El animal presentaba una baja condición corporal, con signos de disnea, eliminando fluido pulmonar a la prueba de la «carretilla». Al examen de necropsia, los pulmones estaban agrandados, renuentes a colapsar, con lesiones neoplásicas nodulares circunscritas en la región cráneo dorsal de los lóbulos diafragmáticos. Las lesiones tenían consistencia dura, de color gris metálico, rodeadas de una fina área blanquecina (Fig. 1B) y con abundante cantidad de una sustancia blanquecina espumosa emanando del ducto traqueal (Fig. 1A). Al corte, el nódulo tumoral evidenciaba una apariencia húmeda compuesta por numerosos micronódulos ligeramente elevados, de contorno irregular, y con tendencia a la coalescencia, así como emanaciones de fluido espumoso del árbol bronquiolar (Fig. 1C). Los nódulos pulmonares regionales estaban agrandados, pero sin mostrar evidencias metastásicas.

Histológicamente, las lesiones fueron de tipo acinar, manteniendo intactas las paredes alveolares pero soportando crecimientos papilares de células columnares o cuboidales con núcleos generalmente uniformes localizados en la región basal y escasas figuras mitóticas (Fig. 1D). En algunas zonas, los alveolos tumorales se observaban rodeados de abundante tejido estromal fibrosado (Fig. $1 \mathrm{E}, 1 \mathrm{~F})$. En otros campos se visualizaban numerosos alveolos con arquitectura celular aparentemente normal, conteniendo abundante cantidad de macrófagos con citoplasma eosinofílico y aparentemente activados (Fig. 1G). Similares lesiones neoplásicas papilares, sostenidas por tejido conectivo, fueron visualizadas; algunas veces, ocluyendo el lumen bronquiolar (Fig. 1H). La prueba de 


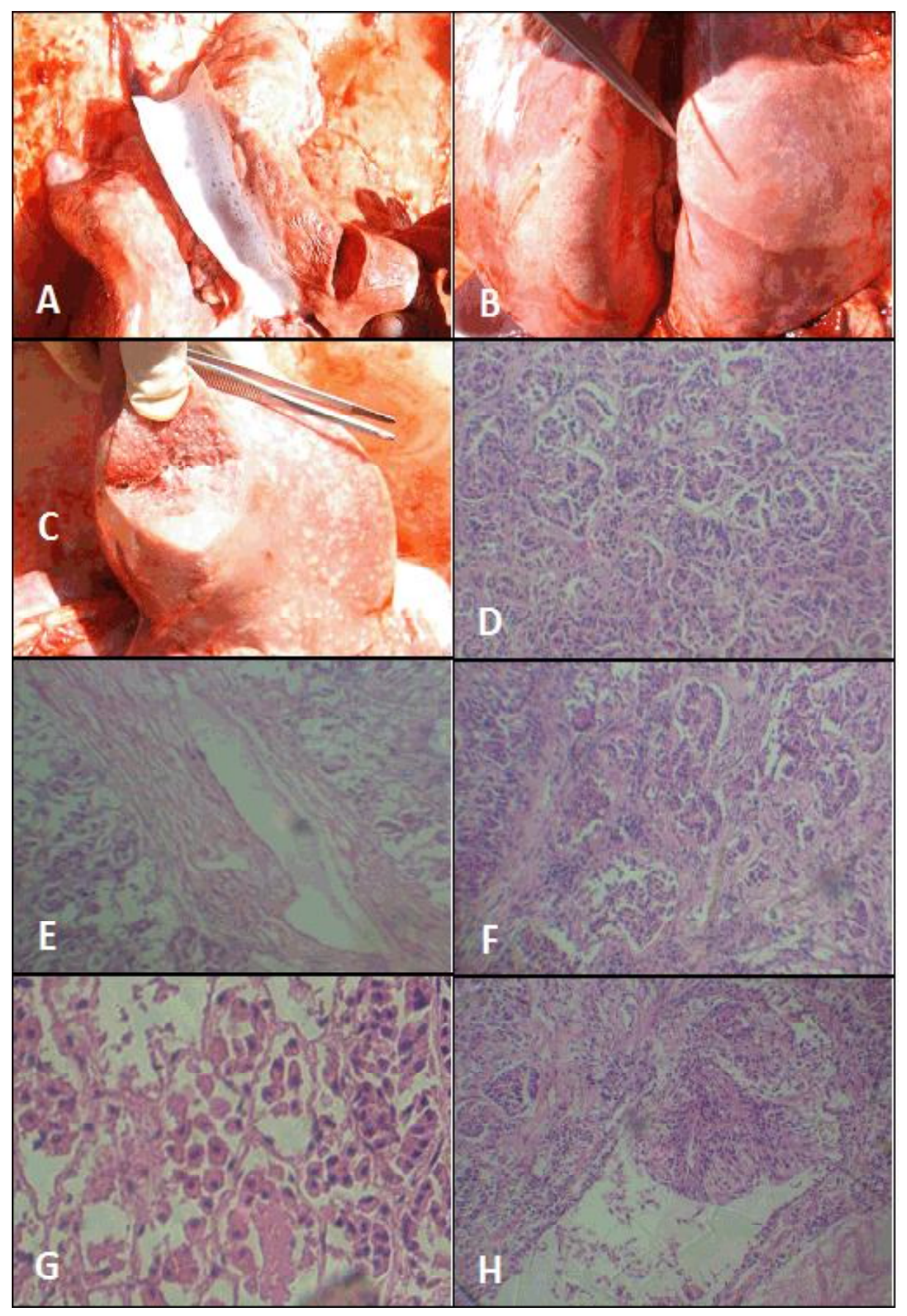

Figura 1. Adenocarcinoma pulmonar ovino en un cordero de 5 meses. A. Presencia de característico líquido blanco espumoso en lumen traqueal; B. Presencia de lesiones neoplásicas nodulares grisáceas blanquecinas en lóbulos diafragmáticos craneales; C. Superficie de corte mostrando numerosos y pequeños nódulos húmedos, ligeramente elevados de contorno irregular con tendencia a la coalescencia, conjuntamente con fluido espumoso brotando de las ramificaciones bronquiales y bronquiolares; $\mathrm{D}$. Nidos tumorales de células columnares reemplazando la arquitectura alveolar normal; E y F. Crecimiento neoplásico asociado a presencia de estroma fibrosado a manera de tabiques; G. Presencia de abundante cantidad de macrófagos alveolares intraluminalmente y rodeando las alteraciones neoplásicas; $\mathrm{H}$. Crecimiento de pólipos neoplásicos intraluminales bonquiolares 
PCR semi-anidada amplificó el ADN proviral de la muestra de tejido tumoral pulmonar.

Las dificultades respiratorias asociadas con eliminaciones de fluidos espumosos observadas en el animal sospechoso y la presencia de lesiones tumorales nodulares con emanaciones de fluido espumoso en las áreas afectadas, así como visualizaciones de acinos compuestos por formaciones papilares de células neoplásicas de tipo columnar o cuboidal en alveolos y bronquiolos, confirman que el caso descrito corresponde a una APO (Cuba Caparó et al., 1961; Nobel et al., 1969; Cutlip y Young, 1982; De las Heras et al., 2003). Asimismo, este diagnóstico fue corroborado con la amplificación de secuencias correspondientes a beta retrovirus exógeno productor de la APO (Palmarini et al., 1996).

Se desconocen sustentos técnicos que expliquen la rápida formación tumoral en corderos, pero se sospecha de la cantidad de dosis infectante y de la inmadurez inmunológica del hospedero. De acuerdo a estudios experimentales, los corderos hasta la novena semana de edad son altamente susceptibles a inoculaciones concentradas, vía intratraqueal, produciendo formaciones tumorales a las 3-4 semanas de la inoculación (Sharp et al., 1983), en tanto que animales mayores de 10 semanas de edad son resistentes a estas inoculaciones (Rosadio et al., 1988).

Las infecciones naturales a temprana edad pueden ser originadas no solamente por exposiciones respiratorias, sino también por ingestiones orales de calostro y leche contaminada (Grego et al., 2008). Este caso es posible que haya sido un evento natural extremadamente raro, pero la presencia de infecciones en animales jóvenes en el sur del Perú bien puede evidenciar una cronología de reciente introducción de la infección retroviral a zonas sureñas, pues la enfermedad, históricamente, ha producido brotes epizoóticos en muchos lugares después de la introducción del virus (York y Querat, 2003).

\section{Literatura Citada}

1. Cuba-Caparó A, De la Vega E, Copaira M. 1961. Pulmonary adenomatosis of sheep. Metastasizing bronchiolar tumours. Am J Vet Res 22: 673-682.

2. Cutlip RC, Young S. 1982. Sheep pulmonary adenomatosis (Jaagsiekte) in the United States. Am J Vet Res 43: 2108-2113.

3. De las Heras M, González L, Sharp JM. 2003. Pathology of ovine pulmonary adenocarcinoma. En: Fan $\mathrm{H}$ (ed). Jaagsiekte sheep retrovirus and lung cancer. New York: Springer. p 25-54.

4. DeMartini JC, Rosadio RH, Lairmore MD. 1988. The etiology and pathogenesis of ovine pulmonary carcinoma (sheep pulmonary adenomatosis). Vet Microbiol 17: 219-236.

5. Ellis JA, Chavera AEV, DeMartini JC. 1993. Disease conditions in slaughtered sheep from small holder flocks in Peru. Small Rumin Res 10: 243-250.

6. González L, Juste RA, Cuervo LA, Idigoras I, Saez de Ocariz C 1993. Pathological and epidemiological aspects of the coexistence of Maedi-Visna and sheep pulmonary adenomatosis. Res Vet Sci 54: 140-146.

7. González L, García-Goti M, Cousens C, Dewar P, Cortabarría N, Extramiana AB, Ortín A, et al. 2001. Jaagsiekte sheep retrovirus can be detected in the peripheral blood during the pre-clinical period of sheep pulmonary adenomatosis. J Gen Virol 82: 1355-1358.

8. Grego E, De Meneghi D, Álvarez V, Benito A, Minguijón E, Ortín A, Mattoni M, et al. 2008. Colostrum and milk can transmit jaagsiekte retrovirus to lambs. Vet Microbiol 130: 247-257.

9. Hunter AR, Munro R. 1983. The diagnosis, occurrence and distribution of sheep pulmonary adenomatosis in Scotland 1875 to 1981 . British Vet J 139: 153-164. 
10. Nobel TA, Neumann F, Klopfer U. 1969. Histological patterns of the metastases in pulmonary adenomatosis of sheep (Jaagsiekte). J Comp Pathol 79: 537-540.

11. Palmarini M, Sharp JM, De las Heras M, Fan H. 1999. Jaagsiekte sheep retrovirus is necessary and sufficient to induce a contagious lung cancer in sheep. J Virol 73: 6964-6972.

12. Palmarini M, Holland MJ, Cousens C, Dalziel RG, Sharp JM. 1996. Jaagsiekte retrovirus establishes a disseminated infection of the lymphoid tissues of sheep affected of pulmonary adenomatosis. J Gen Virol 77: 2991-2998.

13. Paredes T. 1953. Contribución al estudio clínico de la poliadenomatosis pulmonar del carnero. Tesis de Bachiller. Lima: Univ Nacional Mayor de San Marcos. $132 \mathrm{p}$.

14. Rosadio RH, Lairmore MD, Russell HI, DeMartini JC. 1988. Retrovirusassociated ovine pulmonary carcinoma (sheep pulmonary adenomatosis) and lymphoid interstitial pneumonia. I. Lesions development and age susceptibility. Vet Pathol 25: 475-483.

15. Rosadio R, Zavaleta A. 2001. Adenomatosis pulmonar ovina: nuevos conceptos etiopatogénicos Rev Inv Vet Perú 12(Supl 1): 149-153.
16. Sharp JM, DeMartini JC. 2003. Natural history of JSRV in sheep. En: Fan $\mathrm{H}$ (ed). Jaagsiekte sheep retrovirus and lung cancer. New York: Springer. p 55-80.

17. Sharp JM, Angus KW, Gray EW, Scott FM. 1983. Rapid transmission of sheep pulmonary adenomatosis (jaagsiekte) in young lambs. Arch Virol 78: 89-95.

18. Sharp JM, Angus KW. 1990. Sheep pulmonary adenomatosis: clinical, pathological and epidemiological aspects. In: Petursson G, Hoff-Jorgensen R. Maedi-Visna and related diseases. Bostos, USA: Ed. Kluwer Academic Publisher. p 157-175.

19. Snyder SP, DeMartini JC, Ameghino E, Calleti E. 1983. Coexistence of pulmonary adenomatosis and progressive pneumonia in sheep in the central Sierra of Peru. Am J Vet Res 44: 1334-1338.

20. Tustin RC. 1969. Ovine Jaagsiekte. J S Afr Vet Med Assoc 40: 3-23.

21. York DF, Querat G. 2003. History of ovine pulmonary adenocarcinoma (jaagsiekte) and experiments leading to the deduction of the JSRV nucleotide sequence. En: Fan H (ed). Jaagsiekte sheep retrovirus and lung cancer. New York: Springer. p 1-24.

22. Wandera JG 1970. Clinical pulmonary adenomatosis of sheep produced experimentally. Br Vet J 126: 185-193. 